\title{
Numerical Model for Simulation of the Cathodic Protection System with Dynamic Nonlinear Polarization Characteristics
}

\author{
ADNAN MUJEZINOVIĆ, IRFAN TURKOVIĆ, ALIJA MUHAREMOVIĆ \\ Faculty of Electrical Engineering \\ University of Sarajevo \\ Zmaja od Bosne bb, Sarajevo \\ BOSNIA AND HERZEGOVINA
}

\author{
SANJA MARTINEZ \\ Faculty of Chemical Engineering and Technology \\ University of Zagreb \\ Trg Marka Marulića 19, Zagreb \\ CROATIA \\ SLOBODAN MILOJKOVIĆ \\ Faculty of Electrical Engineering \\ University of East Sarajevo \\ BOSNIA AND HERZEGOVINA
}

\begin{abstract}
Cathodic protection is defined as a method for slowing down or complete elimination of corrosion processes on underground or underwater, insulated or uninsulated metal structures. Protection by cathodic protection system is achieved by polarizing protected object to more negative value, with respect to its equilibrium potential. Design of the cathodic protection system implies determination of the electric potential and current density on the electrode surfaces after installation of the cathodic protection system. Most efficient way for determination of the electric potential and current density in the cathodic protection system is by applying numerical techniques. When modeling cathodic protection systems by numerical techniques, electrochemical reactions that occur on electrode surfaces are taken into account by polarization characteristics. Because of nature of the electrochemical reactions, polarization characteristics are nonlinear and under certain conditions can be time - varying (dynamic nonlinear polarization characteristics). This paper deals with numerical modeling of the cathodic protection system with dynamic nonlinear polarization characteristics. Numerical model presented in this paper is divided in the two parts. First part, which is based on the direct boundary element method, is used for the calculation of the distribution of electric potential and current density on the electrode surfaces in the spatial domain. Second part of the model is based on the finite difference time domain method and is used for the calculation of the electric potential and current density change over time. The use of presented numerical model is demonstrated on two simple geometrically examples.
\end{abstract}

Key-Words: - Boundary Element Method, Finite Difference Time Domain Method, Newton-Raphson Technique, Nonlinear Systems

Received: February 16, 2019. Revised: April 2, 2020. Accepted: April 18, 2020. Published: April 30, 2020.

\section{Introduction}

Cathodic protection is most widely used technique for protection of the underground and underwater metallic structures from corrosion [1]. This technique is based on the shifting the equilibrium potential of protected structure to more negative value. This can be done by connecting the protected object with additional electrode(s) placed in same electrolyte (ground or water), which equilibrium potential is more negative than equilibrium potential of the protected object [2]. After installation of the cathodic protection system, electric potential value on the entire surface of protected structure must be lower than minimum protection potential value defined by standards [3]. Also, current density distribution on the surface of protected structure 
should be uniform as possible [4]. Therefore, for evaluation of efficiency of the cathodic protection system, electric potential and protection current density distribution on the protected metallic structure surface need to be known [5]. These parameters can be determined by solving Laplace partial differential equation along with the adequate boundary conditions [1]. This type of the problems can be easily solved by using numerical techniques. For modeling of the cathodic protection systems, boundary element method is mostly used [6-8]. The main advantage of this method, in comparison to the other numerical methods, is that it requires discretization only of boundaries of considered domain and there is no need for discretization of infinite boundaries [9]. Additionally, iterative techniques for solution of nonlinear equations (such as Newton - Raphson technique) must be included because nonlinear boundary conditions (nonlinear polarization characteristics) are given on electrode surfaces of cathodic protection system.

In some situations, these nonlinear boundary conditions can change over the time (dynamic nonlinear polarization characteristics) [10-12]. These time changes of boundary conditions affect the electric potential and current density distribution in the system. Therefore, for modeling of the cathodic protection system with dynamic nonlinear polarization characteristics, some modifications in mathematical model must be done. In this paper, this problem is solved by using combined boundary element method and finite difference time domain method.

\section{Mathematical Model}

As previously mentioned, the purpose of modeling of the cathodic protection system is to determine the distribution of protection current density and electric potential on the surface of the protected structure in order to verify the effectiveness of the system. Electric potential distribution of cathodic protection system can be determined by solving Laplace partial differential equation for static current field:

$$
\nabla^{2} \varphi=0
$$

where $\varphi$ is electric potential and $\nabla$ is Nabla operator.

To obtain a unique solution it is necessary to add the appropriate boundary conditions on domain boundaries to a Laplace partial differential equation. Boundaries that are taken into account when modeling cathodic protection system are anode/electrolyte interface and cathode/electrolyte interface. Boundary conditions applied to these boundaries are called polarization characteristics and they are influenced by electrochemical reaction that takes place on electrode surfaces. Polarization characteristics represent the correlation between current density and electric potential on electrode surfaces and they are nonlinear. When polarization characteristics change over the time then they are dynamic polarization characteristics. These changes of polarization characteristics over the time can be caused by calcareous and magnesium deposition on the electrode surface. This phenomenon is characteristic for the cathode surface $[11,13]$.

Dynamic polarization characteristic on the cathodic surface is defined by the following mathematical relation:

$$
\begin{aligned}
& j_{C}=j_{O_{2}}^{*} f_{O_{2}}+j_{H_{2}}\left(10^{\left(\frac{\varphi-\varphi_{H_{2}}^{*}}{\beta_{H_{2}}}\right)} f_{H_{2}}\right) \\
& -j_{F e}\left(10^{\left(\frac{\varphi-\varphi_{F e}^{*}}{\beta_{F e}}\right)} f_{F e}\right)
\end{aligned}
$$

where $j^{*} O_{2}$ is threshold current density of oxygen reduction, $j_{H_{2}}$ is current density of the hydrogen separation, $j_{F e}$ is current density of the metal dissolution, $\varphi_{H_{2}}^{*}$ and $\varphi_{F e}^{*}$ are equilibrium potentials for corresponding electrochemical reactions, $\beta_{H_{2}}$ and $\beta_{F e}$ are Tafel's coefficients, $\varphi$ is potential difference of interface metal/electrolyte and $f_{\mathrm{O}_{2}}, f_{\mathrm{H}_{2}}$ and $f_{\mathrm{Fe}}$ are time-dependent factors. For all three partial electrochemical reactions, the time-dependent factors have a following form:

$$
f_{R}=e^{-\frac{t}{\tau_{R}}}+f_{\infty, R}\left(1-e^{-\frac{t}{\tau_{R}}}\right)
$$

where index $R$ represents a corresponding electrochemical reaction.

On the anode surface, polarizing characteristic is defined by the following mathematical relation: 


$$
j_{a}=j_{O_{2}}\left(10^{\frac{\varphi-\varphi_{a}}{\beta_{a}}}-1\right)
$$

where $\mathrm{jO}_{2}$ is current density corresponding to the oxygen reduction, $\varphi_{a}$ is equilibrium potential of galvanic anode and $\beta_{a}$ is Tafel slope of galvanic anode.

Graphical representation of the polarization characteristics for anode and cathode surface is given on the Figure1. Polarization characteristics are constructed using relations (2) and (4) and data from literature [13].

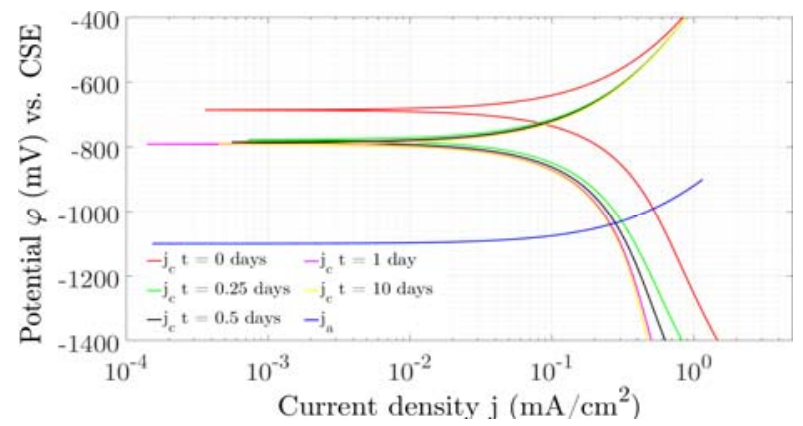

Figure 1. Polarization characteristics of the electrode surfaces

For calculation of the electric potential and current density distribution of the cathodic protection system with dynamic polarization characteristic, numerical procedure based on boundary element method and finite difference time domain method was used. This numerical procedure can be divided into two parts. First part is calculation of electric potential and current density distribution in spatial domain at $t=0$ using boundary element method and second part for calculation of electric potential and current density change over the time using finite difference time domain method.

\subsection{BEM formulation in spatial domain}

Boundary element method for calculation of the electric potential and current density distribution in spatial domain is based on solving the integral field equation. Integral field equation can be obtained by applying Green's symmetric identity on Laplace partial differential equation (1). Then integral field equation have the following form:

$$
\begin{aligned}
& c(q) \varphi(q)+\int_{\Gamma} \varphi(p) \cdot(\vec{n} \cdot \nabla G(p, q)) d \Gamma= \\
& =-\rho \cdot \int_{\Gamma} G(p, q) \cdot j(p) d \Gamma+\varphi_{\infty}
\end{aligned}
$$

where $p$ is position of the source point, $q$ is position of the field point, $\Gamma$ is boundary of the domain, $\varphi(p)$ is electric potential of source point $p, \varphi(q)$ is electric potential of field point $q, c(q)$ is a constant, $\vec{n}$ is normal unit vector, $\rho$ is resistivity of surrounding electrolyte, $j(p)$ is current density at source point $p$, $\varphi_{\infty}$ is constant potential at infinite boundary and $G(p, q)$ is Green's function whose form for 2D plan parallel problems is as follows:

$$
G(p, q)=\frac{1}{2 \pi} \ln \frac{1}{|p-q|}
$$

For problems with infinite boundaries, Gauss boundary condition must be added [14]:

$$
\int_{\Gamma} j(p) d \Gamma=0
$$

In this way it is ensured that there is no flow of current on infinite boundary, and by using Gauss boundary condition there is no need for discretization of infinite boundary.

In the process of calculation of electric potential and current density distribution using boundary element method, it is necessary to discretize domain boundary on appropriate number of boundary elements. After discretization of domain boundaries, field integral equation (5) is solved over every element. After application of the Collocation method at the point and numerical integration, solution of the electric potential and current density can be obtained for each node of all boundary elements by solving following matrix equation [15]:

$$
[H] \cdot\{\varphi\}=[G] \cdot\{j\}
$$

where $\{\varphi\}$ is vector of unknown electric potentials in colocation points, $\{j\}$ is vector of current densities in colocation points, $[H]$ and $[G]$ are matrix of influence coefficients of system geometry.

Since nonlinear boundary conditions are specified, to solve the matrix equation (8) it is necessary to apply an iterative technique. In this paper, matrix equation (8) is solved by using Newton - Raphson iterative technique proposed in $[11,12]$.The first step in solving a matrix equation 
(8) by Newton - Raphson technique is expansion of the vector current density in the Taylor series, as follows:

$$
\{j\}_{k}=\{j\}_{k-1}+\left[\frac{\partial j}{\partial \varphi}\right]_{k-1} \cdot\{\Delta \varphi\}_{k}
$$

where $k$ is number of the current iteration, $[\partial j / \partial \varphi]$ is Jacobian matrix, and $\{\Delta \varphi\}$ is vector of electric potential increments between the two adjacent iteration, and can be written as:

$$
\{\Delta \varphi\}_{k}=\{\varphi\}_{k}-\{\varphi\}_{k-1}
$$

By including matrix equations (9) and (10) in the equation (8), following equation can be written $[11,12]$ :

$$
\left([H]-[G]\left[\frac{\partial j}{\partial \varphi}\right]\right) \cdot\{\Delta \varphi\}_{k}=[G] \cdot\{j\}_{k-1}-[H] \cdot\{\varphi\}_{k-1}
$$

From previous matrix equation vector of electric potential increments in $k-$ th iteration can be calculated. Further, this vector is used in matrix equations (9) and (10) for calculation of current densities and electric potentials in same iteration. Iterative cycle is repeated until a convergence is achieved.

Since this calculation can be time consuming, some modifications are done in this paper in order to accelerate calculation procedure. In matrix equations (9) and (11) Jacobian matrix is used. This matrix is a rectangular matrix whose all elements outside the one diagonal are equal to zero. Therefore, instead of storing a rectangular matrix, non - zero elements of Jacobian matrix are stored in form of vector, as follows:

$$
\left[\begin{array}{cccc}
\frac{\partial j}{\partial \varphi_{11}} & 0 & 0 & 0 \\
0 & {\frac{\partial j}{\partial \varphi_{22}}} & 0 & 0 \\
0 & 0 & {\frac{\partial j}{\partial \varphi_{33}}} & 0
\end{array}\right] \rightarrow\left\{\frac{\partial j}{\partial \varphi}\right\}_{v}=\left\{\begin{array}{l}
\frac{\partial j}{\partial \varphi_{11}} \\
\frac{\partial j}{\partial \varphi_{22}} \\
\frac{\partial j}{\partial \varphi_{33}}
\end{array}\right\}
$$

As the Jacobian matrix has taken a different form, it was necessary to define ways of operating with a given matrix in order to keep its functionality over calculation.

Also, GMRES iterative technique was used for calculation of matrix equation (11) in each iteration of Newton - Raphson method.

\subsection{FTDT formulation in time domain}

The previously given mathematical model represents a solution of the distribution of electrical potential and current density in the cathodic protection system for polarization characteristics of electrode surface at time moment $t=0$. In order to calculate the change of the electric potential and current density over time, previously given mathematical model must be expanded.

Since dynamic (time - varying) polarization characteristics are assumed on cathode surface, change of the current density on this surface can be expressed by expending the function of current density on cathode surface (2) into Taylor series. This can be written as follows:

$$
\left\{j_{c}\right\}_{n}=\left\{j_{c}\right\}_{n-1}+\left[\frac{\partial j_{c}}{\partial \varphi}\right]_{n-1} \cdot\left\{\Delta \varphi_{c}\right\}_{n}+\left[\frac{\partial j_{c}}{\partial t}\right]_{n-1} \cdot\{\Delta t\}_{n}
$$

where $n$ is time step at which current density is calculating, $\left[\partial j_{c} / \partial t\right]$ is matrix of first derivatives of cathode surface current density function with respect to time, $\{\Delta t\}$ is vector of time steps and $\left\{\Delta \varphi_{c}\right\}_{\mathrm{n}}$ is vector of electric potentials increments in $n$ - th time step and can be written as:

$$
\left\{\Delta \varphi_{c}\right\}_{n}=\left\{\varphi_{c}\right\}_{n}-\left\{\varphi_{c}\right\}_{n-1}
$$

From equation (4) it is noticeable that polarization characteristic of anode isn't time dependent. Changes of the electric potential and current density on the anode surface are caused by change of total current intensity of the cathodic protection system caused by dynamic nonlinear polarization characteristic of the cathode surface. Therefore, change of the anode surface current density, caused by the change of the electric potential in the $n$-th time step can be expressed as follows:

$$
\left\{j_{a}\right\}_{n}=\left\{j_{a}\right\}_{n-1}+\left[\frac{\partial j_{a}}{\partial \varphi}\right]_{n-1} \cdot\left\{\Delta \varphi_{a}\right\}_{n}
$$

where $\left[\partial j_{a} / \partial t\right]$ is matrix of first derivatives of anode surface current density function with respect to time, $\left\{\Delta \varphi_{a}\right\}_{\mathrm{n}}$ is vector of electric potentials increments in $n$-th time step and can be written as:

$$
\left\{\Delta \varphi_{a}\right\}_{n}=\left\{\varphi_{a}\right\}_{n}-\left\{\varphi_{a}\right\}_{n-1}
$$


It is important to emphasize that Gauss condition must be satisfied at each time step. Taking into account all the above, the overall matrix equation can be written in the following form:

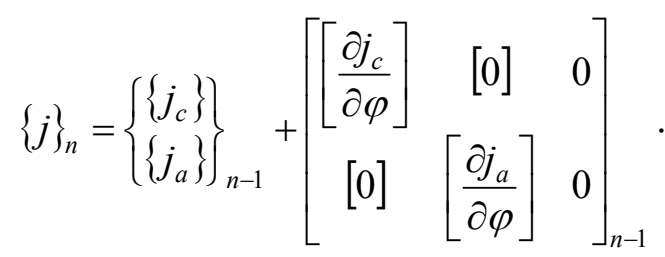

$$
\begin{aligned}
& \cdot\left\{\begin{array}{c}
\left\{\Delta \varphi_{c}\right\} \\
\left\{\Delta \varphi_{a}\right\} \\
0
\end{array}\right\}_{n}+\left\{\begin{array}{c}
\left\{\frac{\partial j_{c}}{\partial t}\right\} \\
\{0\} \\
0
\end{array}\right\}_{n-1} \cdot \Delta t
\end{aligned}
$$

The matrix equation (17) defines the current density of the cathodic protection system in the $n$ th time step. Due to the equation dimensionality, additional vector is added to the vector of derivatives of current density in the time. The elements of the added vector are all equal to zero and the length of this vector is equal to the number of collocation points on the surface of the anode. Also, in matrix equation (17), to matrix of current density derivatives with respect to the time and vector of potential increments another element equal to zero is added. Objective of this is satisfaction of Gauss boundary conditions.

In matrix equation (17) two vectors are unknown. By including matrix equations (14), (16) and (17) in the matrix equation (8), the following matrix equation with one unknown vector can be obtained:

$$
\begin{aligned}
& \left.\left([H]-[G] \frac{\partial j}{\partial \varphi}\right]_{n-1}\right) \cdot\{\Delta \varphi\}_{n}=[G] \cdot\{j\}_{n-1}+ \\
& +[G] \cdot\left[\frac{\partial j}{\partial t}\right]_{n-1}\{\Delta t\}_{n}-[H] \cdot\{\varphi\}_{n-1}
\end{aligned}
$$

By solving matrix equation (18) vector of electric potential increments in $n$ - th time step is obtained. Further, this vector is used in matrix equations (14), (16) and (17) for calculation of current densities and electric potentials in the same time step.

\subsection{Calculation of current intensity}

Since cathodic protection system is a conserved system (i.e. all current that leaves the anode enters into the cathode), current intensity can be calculated by using the following equation:

$$
I=\Gamma_{c} \cdot j_{c}=\Gamma_{a} \cdot j_{a}
$$

where $\Gamma_{c}$ is the surface of the cathode, $\Gamma_{a}$ is the surface of the anode, $j_{c}$ is the current density of the cathode surface and $j_{a}$ is the current density of the anode surface.

Current density, in most practical cases, is not homogeneously distributed on both cathode and anode surface. Therefore, equation (19) is not suitable to use for the calculation of current intensity of cathodic protection system in this form. This problem can be overcome by using the calculated current densities of collocation points and their associated surfaces. Current intensity of the galvanic anode cathodic protection system, after discretization and after calculation of current density distribution in the cathodic protection system, can be calculated by the following equation [16]:

$$
I_{n}=\left\{A_{c}\right\} \cdot\left\{j_{c}\right\}_{n}=\left\{A_{a}\right\} \cdot\left\{j_{a}\right\}_{n}
$$

where $\left\{A_{c}\right\}$ and $\left\{A_{a}\right\}$ are column matrix of coefficients that correspond to surfaces of colocation points. The elements of these matrices are contained in the last row of the matrix $[\mathrm{G}]$ in the equation (8). For the closed region, elements of these matrices must be calculated separately.

\subsection{Calculation of driving voltage}

For simple geometries, driving voltage of the cathodic protection system can easily be calculated, as well as its change over time (defined as the difference between values of potentials on the cathode and anode surface). Driving voltage can be calculated by using the following equation:

$$
U_{d n}=\varphi_{c, n}-\varphi_{a, n}
$$

where $\varphi_{c, n}$ is the value of electric potential in one point of cathode in $n$ - th time step, $\varphi_{a, n}$ is the value of electric potential in one point of anode in $n$ - th time step and $U_{d, n}$ is voltage drop in $n$ - th time step.

The unique value of the voltage drop can be calculated only for cathodic protection system with homogeneous distribution of the electric potential (rare case in the practical situations). 


\section{Application of the proposed model}

Application of the previously presented mathematical model is demonstrated on two examples. For both cases radius of anodes is $1 \mathrm{~m}$ while radius of cathode is $5 \mathrm{~m}$. Also, for all cases resistivity of the electrolyte is $25 \Omega \mathrm{cm}$.

\subsection{Case Study 1}

Calculation results of distribution of the electric potential and current density for Case Study 1, are presented below. Geometry of the Cases Study 1 is shown on Figure 2.

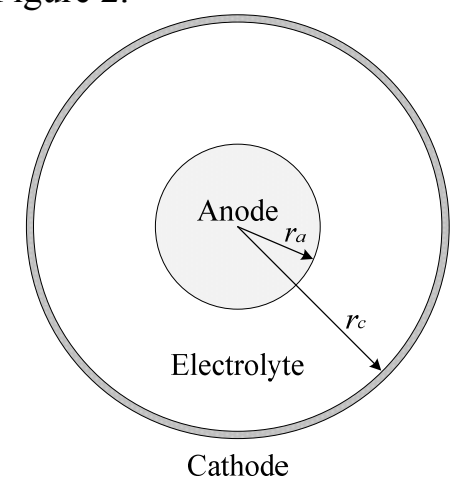

Figure 2. Geometry of analyzed galvanic anode catohdic protection system

Due to the symmetry of the analyzed system, the distribution of the electric potential and current density is uniform. Therefore, it is enough to present the results of a calculation in a single point on the surfaces of the anode and cathode. Distribution of the electric potential on the cathode surface is given on the Figure 3.

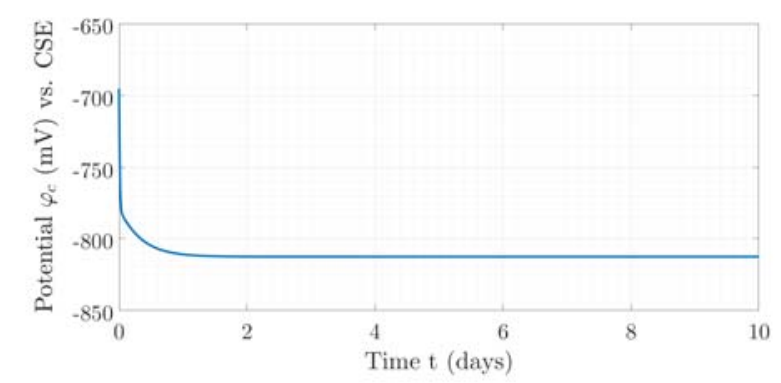

Figure 3. Electric potential distribution on the cathode surface over time

From results given on the Figure 3 it can be noted that electric potential on the cathode surface is changing over time. Electric potential of the cathode surface becomes more negative over time, which further contributes to the protection against corrosion.
Change of the current density distribution on the cathode surface over the time is given on the Figure 4.

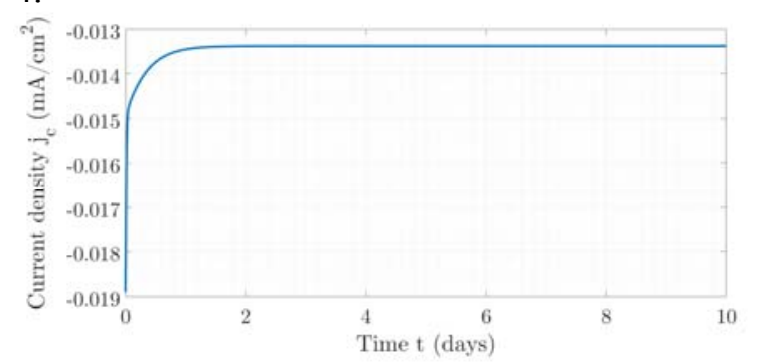

Figure 4. Current density distribution on the cathode surface over time

From the results given on the Figure 4 it can be noted that requirement for the current density of the cathode surface is reduced over time.

Distribution of the electric potential and current density on the anode surface over the time is given on the Figures 5 and 6, respectively.

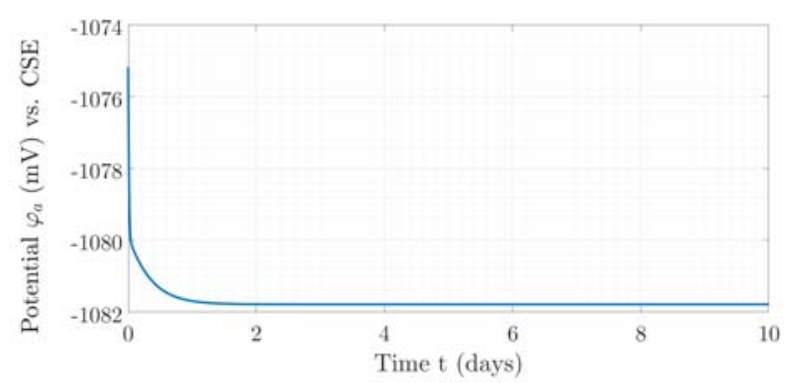

Figure 5. Electric potential distribution on the anode surface over time

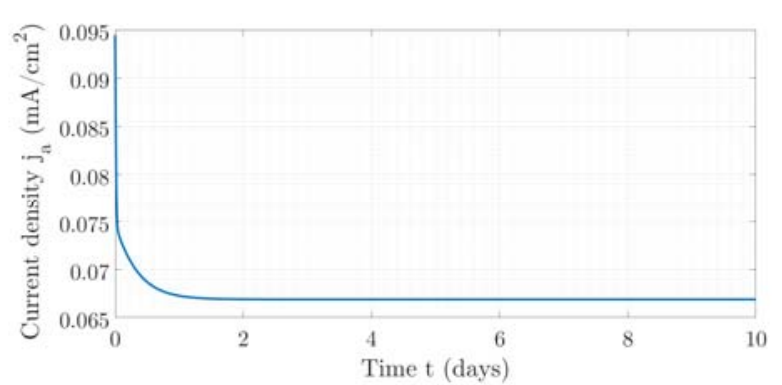

Figure 6. Current density distribution on the anode surface over time

From results given on the Figures 5 and 6 it is noticeable that electric potential becomes more negative over time while the current density of anode surface decreases over time. Decrease of the current density on the anode surface causes a reduction of anode material consumption, which also contributes to the protection against corrosion.

On Figure 7, change of the current intensity of the cathodic protection system over time is given. 


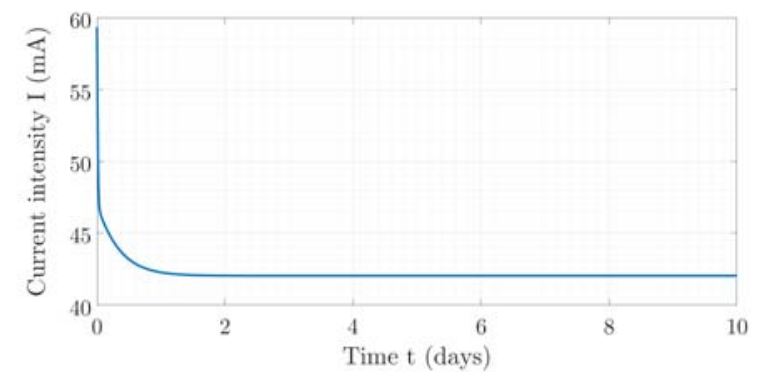

Figure 7. Change of the current intensity of $\mathrm{CP}$ system over time

From the results given on the Figure 7 it can be noted that current intensity also decreases over time.

Since electric potential distribution on both electrodes (anode and cathode) is uniform, unique value of the driving voltage can be calculated. Change of the driving voltage for the analyzed cathodic protection system is given on the Figure 8.

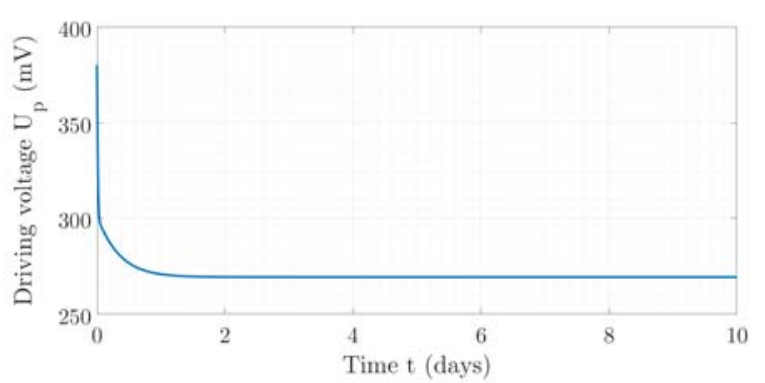

Figure 8. Change of the driving voltage of $\mathrm{CP}$ system over time

From results given on the Figure 8 it can be noted that driving voltage decreases over the time. Although, driving voltage represents difference of anode and cathode potential which both change over time on negative side, but rate of change are different. Therefore, driving voltage also change over time.

\subsection{Case Study 2}

In Case Study 2 anode is placed outside of the cathode. Both electrodes are placed in the infinite electrolyte. Distance between centers of the electrodes is $d=5 \mathrm{~m}$. Geometry of the analyzed cathodic protection system is given on the Figure 9.

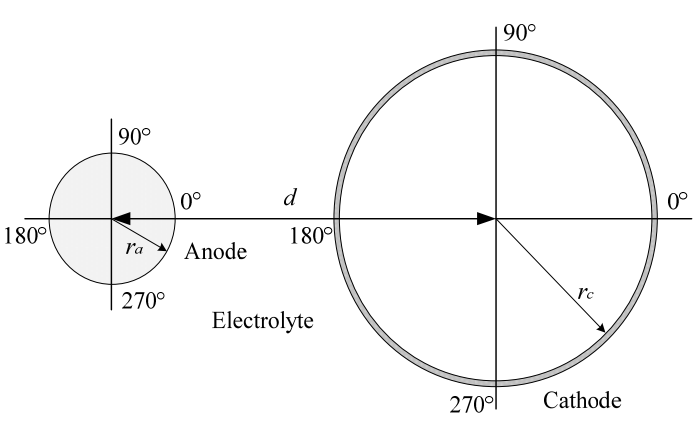

Figure 9. Geometry of analyzed galvanic anode catohdic protection system

Results of the calculation of electric potential and current density on the cathode and anode surface are presented below. Distribution of electric potential on the cathode surface is given on the Figure 10.

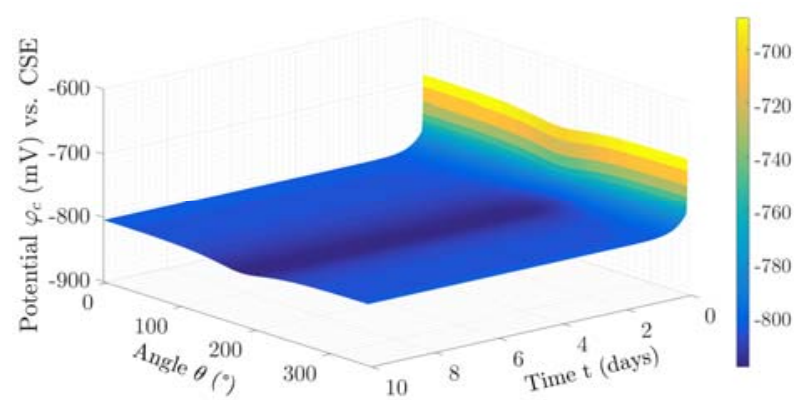

Figure 10. Change of electric potential distribution on the cathode surface over time

Results given on the Figure 10 indicate that areas that are closer to the anode (the nearest point is located at $180^{\circ}$ ) have lower values of electric potential. This means that this area is more protected than the other areas of the cathode. Also, it can be noticed that the electric potential becomes more negative in all points of the cathode, which supports the cathodic protection of surfaces against corrosion. Change of the current density distribution on the cathode surface in the discrete time steps is given on the Figure 11.

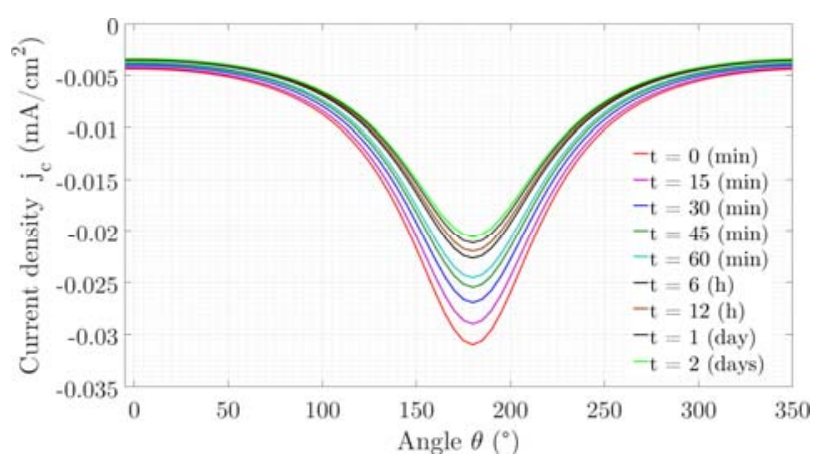

Figure 11. Current density distribution on the cathode surface over time 
From results given on the Figure 4 it is noticeable that the current density is higher (in absolute sense) at areas that are closer to the anode. Also, increase of the current density over the time (also in absolute sense) is higher in areas that are closer to the anode.

Electric potential and current density distribution on the anode surface over the time are given on the Figures 12 and 13, respectively.

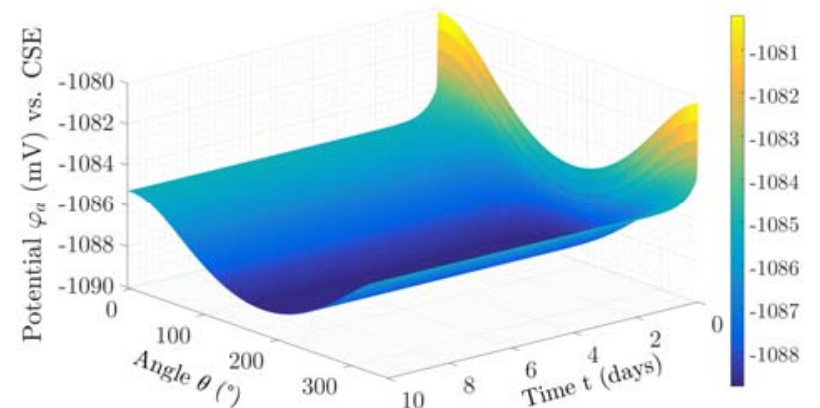

Figure 12. Electric potential distribution on the anode surface over time

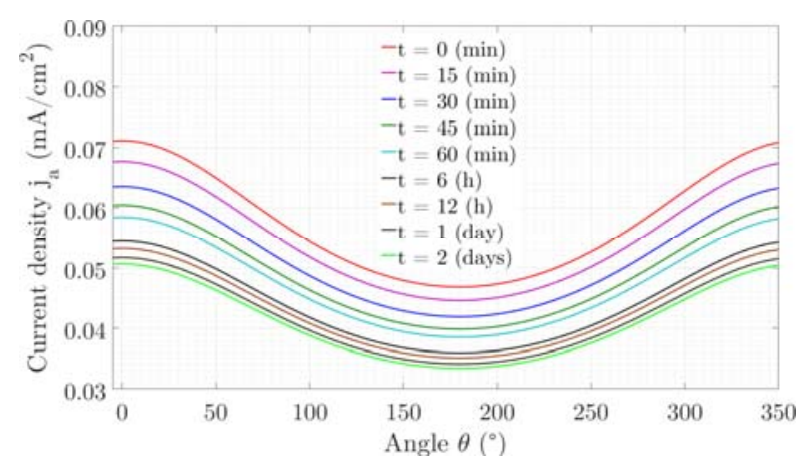

Figure 13. Current density distribution on the anode surface over time

It can be noticed that the electrical potential on anode surface is more positive on areas where the anode surface is closer to the cathode (the nearest point is located at $0^{\circ}$ ). Also, on this areas current density has the highest values.

Change of the current intensity in analyzed cathodic protection system, caused by dynamic polarization characteristics is given on the Figure 14.

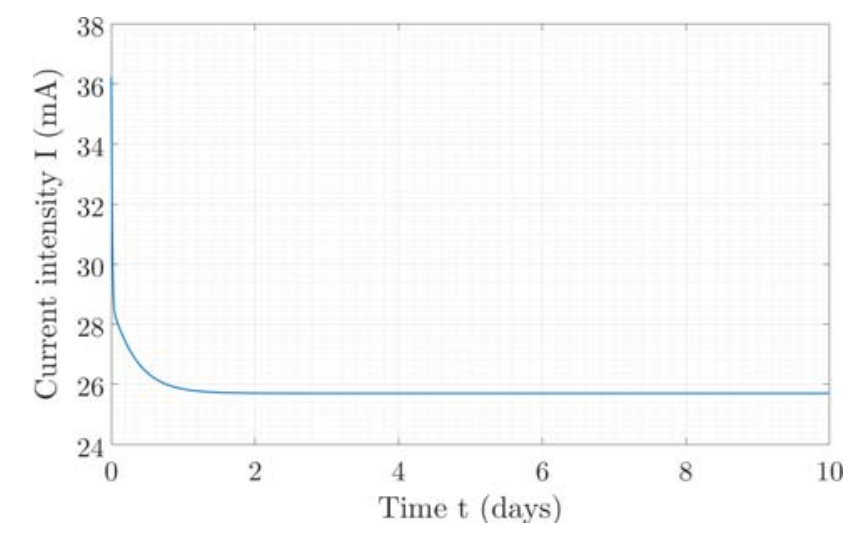

Figure 14. Change of the current intensity of $\mathrm{CP}$ system over time

From results given on the Figure 14 it can be noted that current intensity decreases over the time. Also, highest change of the current intensity is at first day, while after two days current intensity is constant.

\section{Conclusion}

Application of very accurate and precise numerical methods is required for the design of modern cathodic protection system. The main reason for this is the fact that the boundary conditions on the electrode surface of the system are nonlinear and in some situations, such as deposition of calcareous and magnesium on the cathode surface, and may be time-varying (dynamic). In this paper, mathematical model for calculation of the electric potential and current density distribution in galvanic anode cathodic protection system with dynamic nonlinear polarization characteristics is presented. Presented mathematical model is based on combination of boundary element method and finite difference time domain method. Application of presented mathematical model was demonstrated on one 2D simple geometry problem.

\section{Acknowledgment}

This paper was supported by the Ministry of Education and Science of the Federation of Bosnia and Herzegovina.

\section{References:}

[1] L. Lazzari, P.Pedeferri, Cathodic Protection, $1^{\text {st }}$ edition, Polipress Milano, 2006.

[2] B. Jarić, A. Rešetić, Corrosion: Electrochemical fundamentals and cathodic protection, Korexpert, Zagreb, 2003.

[3] W. J. Santos, J. A. F. Santiago, J. C. F. Telles, Using the Gaussian function to simulate constant potential anodes in multiobjective 
optimization of cathodic protection systems, Engineering Analysis with Boundary Elements, Vol. 73, 2016, pp. 35-41.

[4] A. Muharemović, H. Zildžo, E. Letić, Modelling of protective potential distribution in a cathodic protection system using a cupled $\mathrm{BEM} / \mathrm{FEM}$ method, $30^{T H}$ International conference on Boundary Elements Method and Other Reduction Methods, BEM/MRM 30, Maribor, Slovenia, 2008.

[5] I. Turković, Contribution to the study of the influence of nonlinear voltage and current distribution to optimization of the protection length in cathodic protection domain, $\mathrm{PhD}$ Dissertation, Sarajevo 2010.

[6] C. Liu, A. Shankar, M. E. Orazem, P. D. Riemer, Numerical simulations for cathodic protection of pipelines, in Underground pipeline corrosion - Detection, analysis and prevention, Edited by M. E. Orazem, Woodhead Publishing, 2014.

[7] J. F. Fan, S. N. R. Pakalapati, T. V. Nguyen, R. E. White, R. B. Griffin, Mathematical Modelling of Cathodic Protection Using the Boundary Element Method with a Nonlinear Polarization Curve, Journal of The Electrochemical Society, Vol. 139, No. 7, 1992, pp. 1932-1936.

[8] A. Mujezinović, A. Muharemović, I. Turković, A. Muharemović, Calculation of the Protective Current Density Distribution of a Cathodic Protection System with Galvanic Anodes in Terms of Double-layer Electrolyte, $34^{T H}$ International conference on Boundary Elements Method and Other Reduction Methods, BEM/MRM, Croatia, Split, 2012.

[9] S. Martinez, Evaluation of the Uniform Current Density Assumption in Cathodic Protection System with Close Anode to Cathode Arrangement, Materials and Corrosion, Vol. 61, No. 4, 2010, pp. $338-342$.

[10] K. Nisancioglu, Predicting the Time Dependence of Polarization on Cathodically Protected Steel in Seawater, Corrosion, Vol. 43, No. 2, 1987, pp. 100 - 111.

[11] J. A. F. Santiago, J. C. F. Telles, On Boundary Elements for Simulation of Cathodic Protection System with Dynamic Polarization Curves, International Journal for Numerical Methods in Engineering, Vol. 40, No. 14, 1997, pp. $2611-2627$.

[12] J. A. F. Santiago, J. C. F. Telles, A Solution Technique for Cathodic Protection System with Dynamic Boundary Conditions by the Boundary Element Method, Advances in engineering software, Vol. 340, No. 9, 1999, pp. $663-671$.

[13] S. L. Carson, M. E. Orazem, Time - dependent polarization behaviour of pipeline grade steel in low ionic strength environments, Journal of Applied Electrochemistry, Vol. 29, No. 6, 1999, pp. $707-721$.

[14] C. A. Brebbia, J. C. F. Telles, L. C. Wrobel, Boundary Element Techniques Theory and Applications in Engineering, Berlin / Heidelberg, Springer-Verlag, 1984.

[15] A. Mujezinović, I. Turković, S. Martinez, S. Milojković, Modelling of the Cathodic Protection System with Dynamic Non-Linear Polarization Characteristics, $X X V$ International Conference on Information, Communication and Automation Technologies (ICAT), Sarajevo 2015.

[16] A. Mujezinović, Modeling of the Galvanic Anode Cathodic Protection System with Dynamic Nonlinear Polarization Characteristics, $\mathrm{PhD}$ Dissertation, Sarajevo 2017.

[17] Raffaele D'Ambrosio, Martina Moccaldi, Beatrice Paternoster, Adapted IMEX Numerical Methods for Reaction-Diffusion Problems, International journal of circuits, systems and signal processing, pp.507-515, Volume 13, 2019 